Electronic Supporting Information (ESI)

\title{
An Organofunctionalized Polyoxovanadium Cluster as a Molecular Model of Interfacial Pseudocapacitance
}

Eric Schreiber ${ }^{\# 1}$, Niamh A. Hartley ${ }^{\# 1}$, William W. Brennessel ${ }^{1}$, Timothy R. Cook ${ }^{2}$, James R. McKone $^{*}$, and Ellen M. Matson ${ }^{* 1}$

\# Denotes authors contributed equally

${ }^{1}$ Department of Chemistry, University of Rochester, Rochester NY 14627, USA

2 Department of Chemistry, University at Buffalo, The State University of New York, Buffalo NY 14260, USA

${ }^{3}$ Department of Chemical and Petroleum Engineering, University of Pittsburgh, Pittsburgh PA 15260, USA

Corresponding Author Contact Information:

Ellen M. Matson: matson@chem.rochester.edu (e-mail)

James R. McKone: imckone@pitt.edu (e-mail)

Table of Contents:

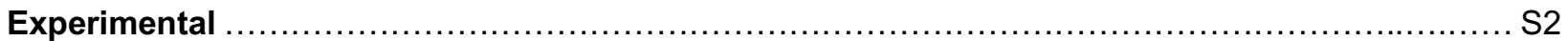

Figure S1. Electrochemical analysis of $\mathrm{R} 2$ for $\mathbf{1}-\mathrm{V}_{6} \mathrm{O}_{7}$ with $\mathrm{LiOTf}$ as a supporting electrolyte.............S4

Table S1. Substoichiometric Electrochemical Titration data ................................................. 55

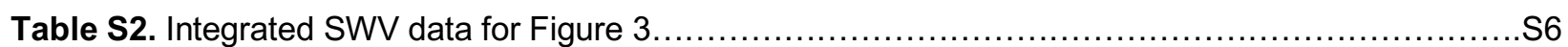

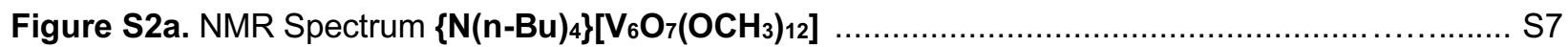

Figure S2b. NMR Spectrum $\left\{\mathrm{N}(\mathrm{n}-\mathrm{Bu})_{4}\right\}\left[\mathrm{V}_{6} \mathrm{O}_{7}\left(\mathrm{OCCH}_{3}\right)_{12}\right]$ from cation exchange reaction .................... S7

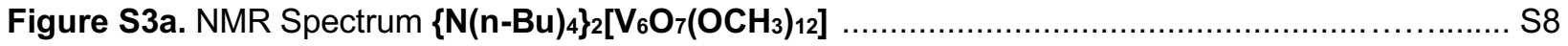

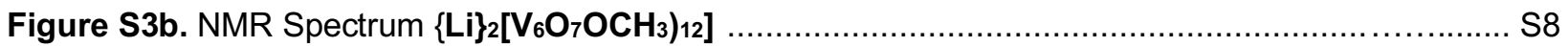

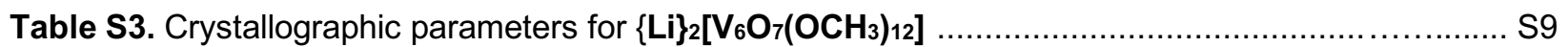

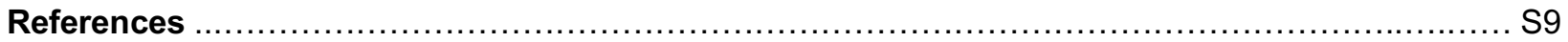




\section{Experimental}

General Considerations. Where specified, air- and moisture-sensitive manipulations were carried out in the absence of water and oxygen in a UniLab MBraun inert atmosphere glovebox under a dinitrogen atmosphere. Glassware was oven dried for a minimum of 4 hours and cooled in an evacuated antechamber prior to use in the glove box. Anhydrous methanol was purchased from Sigma-Aldrich and stored over activated $4 \AA$ molecular sieves (Fisher Scientific). Acetonitrile (Fisher Chemical) used for electrochemical experiments was deoxygenated and dried on a Glass Contour System (Pure Process Technology, LLC) and stored over activated $4 \AA$ molecular sieves (Fisher Scientific). All other solvents (acetone and diethyl ether) were used as received outside the glove box without drying or degassing. $\left\{{ }^{n} \mathrm{Bu}{ }_{4} \mathrm{~N}\right\} \mathrm{OTf}$ and $\left\{{ }^{n} \mathrm{Bu}_{4} \mathrm{~N}_{\mathrm{P}} \mathrm{PF}_{6}\right.$ were purchased from Sigma-Aldrich, recrystallized three times from hot ethanol, and stored under dynamic vacuum for at least one day prior to use. LiOTf, NaOTf, and KOTf were purchased from Sigma Aldrich and used as received. Complexes $\left[\mathrm{V}_{6} \mathrm{O}_{7}\left(\mathrm{OCH}_{3}\right)_{12}\right]$, $\left\{{ }^{\mathrm{n}} \mathrm{Bu} 4 \mathrm{~N}\right\}\left[\mathrm{V}_{6} \mathrm{O}_{7}\left(\mathrm{OCH}_{3}\right)_{12}\right]$, and $\left\{{ }^{\mathrm{n}} \mathrm{Bu} \mathrm{u}_{4} \mathrm{~N}\right\}_{2}\left[\mathrm{~V}_{6} \mathrm{O}_{7}\left(\mathrm{OCH}_{3}\right)_{12}\right]$ were synthesized according to literature procedures. $^{[1]}$

${ }^{1} \mathrm{H}$ NMR spectra were recorded at $400 \mathrm{MHz}$ on a Bruker DPX-400 spectrometer at 9.4 Tesla while locked on to deuterated solvent. Deuterated $\mathrm{C}_{3} \mathrm{H}_{6} \mathrm{O}$ and $\mathrm{CH}_{3} \mathrm{CN}$ were purchased from Cambridge Isotope Laboratories and used as received from the manufacturer. Elemental analysis was performed by Midwest Microlabs (Indianapolis, Indiana). X-ray quality single crystals were mounted on a thin Nylon loop and positioned on a XtaLab Synergy-S Dualflex diffractometer equipped with a HyPix-6000He HPC area detector for data collection at $100.0(5) \mathrm{K}$. Structure solutions were obtained using SHELXT-2014/5 $5^{[2]}$ and refined using SHELXL-2014/7 ${ }^{[3]}$.

Electrochemical Analysis (Cyclic \& Square Wave Voltammetry). For all cyclic voltammetry (CV) experiments, concentrations of active species (vanadium cluster) and all supporting electrolytes

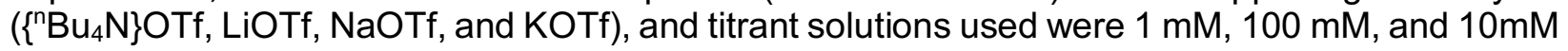
respectively. CV measurements were carried out using a Bio-Logic SP 150 potentiostat/galvanostat and the EC-Lab software suite. Glassy carbon discs $(3 \mathrm{~mm}, \mathrm{CH}$ Instruments, USA) were used as working electrodes. Working electrodes were polished using a micro cloth pad and $0.05 \mu \mathrm{M}$ alumina powder. Potentials recorded during $\mathrm{CV}$ were measured relative to a nonaqueous $\mathrm{Ag} / \mathrm{Ag}+$ reference electrode with $10 \mathrm{mM} \mathrm{AgNO}_{3}$ and $100 \mathrm{mM}$ $\left\{{ }^{n} \mathrm{Bu}_{4} \mathrm{~N}\right\}\left\{\mathrm{PF}_{6}\right\}$ in acetonitrile (Bio-Logic). A platinum wire served as the counter electrode. All experiments were carried out at room temperature inside a nitrogen-filled glove box (MBraun, USA). All CV measurements were iR compensated at $85 \%$ with impedance taken at $100 \mathrm{kHz}$ using the ZIR tool included with the EC-Lab software. CV experiments were conducted at 100 $\mathrm{mV} / \mathrm{s}$. All concentrations, hardware, software, electrodes, reference, and atmospheric parameters for square wave voltammetry experiments were the same as for cyclic voltammetry. Square wave experiments were conducted at $50 \mathrm{mV} / \mathrm{s}$ (pulse height: $25 \mathrm{mV}$, pulse width: $100 \mathrm{~ms}$, step height: $10 \mathrm{mV})$.

Preparation of $\left\{\mathrm{Li}_{2}\left[\mathrm{~V}_{6} \mathrm{O}_{7}\left(\mathrm{OCH}_{3}\right)_{12}\right]\right.$. A $20 \mathrm{~mL}$ scintillation vial was charged with $\left\{{ }^{\mathrm{n} B{ }_{4}} \mathrm{~N}_{2}\left[\mathrm{~V}_{6} \mathrm{O}_{7}\left(\mathrm{OCH}_{3}\right)_{12}\right](0.074 \mathrm{~g}, 0.058 \mathrm{mmol})\right.$ and $5 \mathrm{~mL}$ of acetonitrile. To this solution was added three equivalents of LiOTf $(0.027 \mathrm{~g}, 0.174 \mathrm{mmol})$, weighed by difference. The blue solution was stirred for 20 minutes, during which a blue powder precipitated from the reaction mixture. The reaction was filtered over a glass fritted funnel and the blue powder was washed, three times with acetonitrile $(2 \mathrm{~mL})$ and three times with diethyl ether $(4 \mathrm{~mL})$. The solid was dried under reduced pressure for 1 hour, resulting in isolation of the product as a light blue powder $(0.0402 \mathrm{~g}, 0.050$ $\mathrm{mmol}, 86 \%$ ). Crystals suitable for X-ray analysis were grown from slow diffusion of diethyl ether 
into a concentrated solution of the product dissolved in acetone. After four days, teal blue needles suitable for crystallographic analysis were obtained. ${ }^{1} \mathrm{H}$ NMR $\left(400 \mathrm{mHz},\left(\mathrm{CD}_{3}\right) \mathrm{CO}\right): \delta=21.83 \mathrm{ppm}$ (fwhh $=640 \mathrm{~Hz}$ ). Elemental Analysis for $\mathrm{C}_{12} \mathrm{H}_{36} \mathrm{O}_{19} \mathrm{~V}_{6} \mathrm{Li}_{2}(\mathrm{MW}=803.93 \mathrm{~g} / \mathrm{mol}$ ) Calcd (\%): C, 17.93; $\mathrm{H}, 4.51$; Found (\%): C, 17.88; $\mathrm{H}, 4.63$.

a)
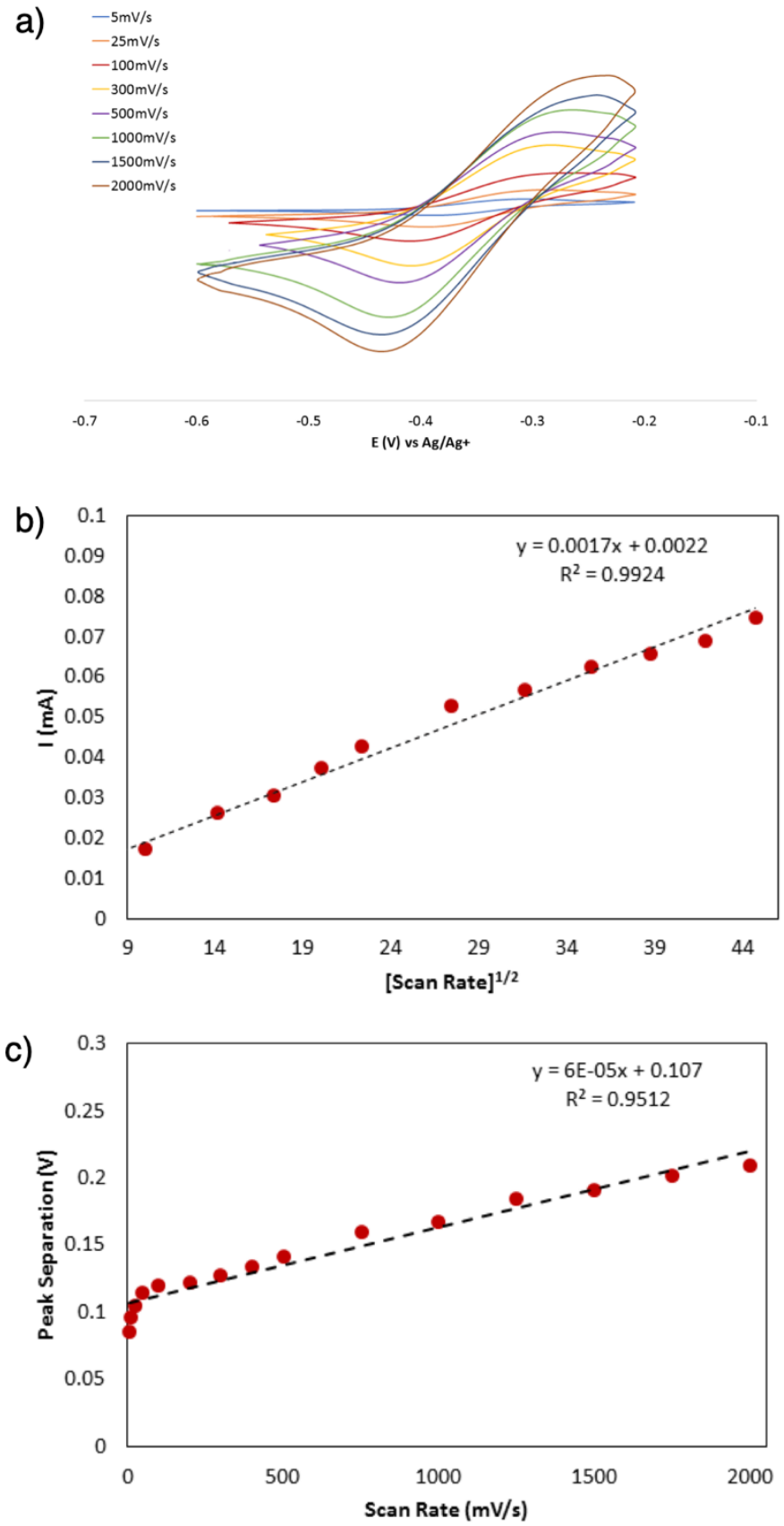
Figure S1. Electrochemical data to test the reversibility of the most reducing redox couple (R2) for complex $\mathbf{1}-\mathbf{V}_{6} \mathbf{O}_{7}$. (a) Cyclic voltammogram of complex $\mathbf{1}-\mathbf{V}_{6} \mathbf{O}_{7}(1 \mathrm{mM})$ taken at scan rates ranging from 5 to $2000 \mathrm{mV} \mathrm{s}^{-1}$ in $\mathrm{MeCN}$ with $0.1 \mathrm{M} \mathrm{LiOTf}$ as the supporting electrolyte; (b) plot of the current density $\left(j_{p}\right)$ versus the square root of the scan rate $\left(n^{1 / 2}\right)$ for complex $1-V_{6} \mathbf{O}_{7}(c)$ plot of peak separation $(\mathrm{V})$ vs. scan rate $(\mathrm{mV} / \mathrm{s})$ for complex $\mathbf{1 - \mathbf { V } _ { 6 }} \mathbf{O}_{7}$.

Table S1. Substoichiometric electrochemical titration of alkali salts into $\mathbf{V}_{6} \mathbf{O}_{7}\left(\mathbf{O C H}_{3}\right)_{12}$ analyzed by $\mathrm{CV}$ and SWV.

\begin{tabular}{|c|c|c|c|c|}
\hline \multicolumn{5}{|c|}{ KOTf } \\
\hline \multirow{2}{*}{\multicolumn{2}{|c|}{$\begin{array}{c}\text { R1 } \\
\text { E (V) vs Ag/Ag+ }\end{array}$}} & \multirow{2}{*}{\multicolumn{3}{|c|}{$\begin{array}{c}\text { R2 } \\
E(V) \text { vs } \mathrm{Ag} / \mathrm{Ag}+\end{array}$}} \\
\hline & & & & \\
\hline Equiv & SWV & Equiv & CV & SWV \\
\hline & -0.1906 & 0 & -0.701 & -0.699 \\
\hline 0.2 & -0.1905 & 0.2 & -0.6944 & -0.6915 \\
\hline 0.5 & -0.1906 & 0.5 & -0.6884 & -0.6816 \\
\hline & -0.1905 & 1 & -0.6804 & -0.6715 \\
\hline & -0.1905 & & -0.6745 & -0.665 \\
\hline
\end{tabular}

\begin{tabular}{rrr|rrr}
\hline \multicolumn{4}{c}{ R1 } & \multicolumn{4}{c}{ NaOTf } \\
Equiv & \multicolumn{2}{c}{ C (V) vs Ag/Ag+ } & \multicolumn{3}{c}{ E (V) vs Ag/Ag+ } \\
\hline 0 & -0.1945 & -0.2006 & & -0.6923 & -0.7018 \\
0.2 & -0.1928 & -0.197 & 0.2 & -0.6708 & -0.6717 \\
0.5 & -0.19 & -0.1905 & 0.5 & -0.6492 & -0.6316 \\
1 & -0.1895 & -0.1905 & & -0.6261 & -0.6116 \\
2 & -0.1879 & -0.1906 & & -0.6065 & -0.6015
\end{tabular}

\begin{tabular}{|c|c|c|c|c|c|c|c|c|}
\hline \multicolumn{9}{|c|}{ LiOTf } \\
\hline \multicolumn{3}{|c|}{$\begin{array}{c}\text { R1 } \\
\text { E (V) vs Ag/Ag+ }\end{array}$} & \multicolumn{3}{|c|}{$\begin{array}{l}\text { R1.5 } \\
\text { E (V) vs } \mathrm{Ag} / \mathrm{Ag}+\end{array}$} & \multicolumn{3}{|c|}{$\begin{array}{c}R 2 \\
E(V) \text { vs } \mathrm{Ag} / \mathrm{Ag}+\end{array}$} \\
\hline Equiv & CV & SWV & Equiv & CV & SWV & Equiv & CV & SWV \\
\hline & -0.1973 & -0.2008 & 0 & - & - & 0 & -0.6977 & -0.7018 \\
\hline 0.2 & -0.191 & -0.1907 & 0.2 & - & -0.5014 & 0.2 & -0.693 & -0.698 \\
\hline 0.5 & -0.1919 & -0.1907 & $0.5-$ & 0.4887 & -0.4814 & 0.5 & -0.6657 & - \\
\hline & -0.1879 & -0.186 & & 0.4748 & -0.4513 & 1 & - & - \\
\hline & -0.1813 & -0.1807 & & 0.4539 & -0.4413 & 2 & - & - \\
\hline
\end{tabular}


Table S2. Integrated SWV data for Figure 3.

\begin{tabular}{rll}
\hline \multicolumn{3}{c}{ SWV Integrated } \\
\multicolumn{3}{c}{ Area Ratio (R2/R1) } \\
\hline Equiv & LiOTf & NaOTf \\
\hline 0 & 0.953 & 0.969 \\
0.2 & 0.998 & 0.976 \\
0.5 & 0.894 & 0.934 \\
1 & 0.906 & 0.957 \\
2 & 0.893 & 0.958
\end{tabular}




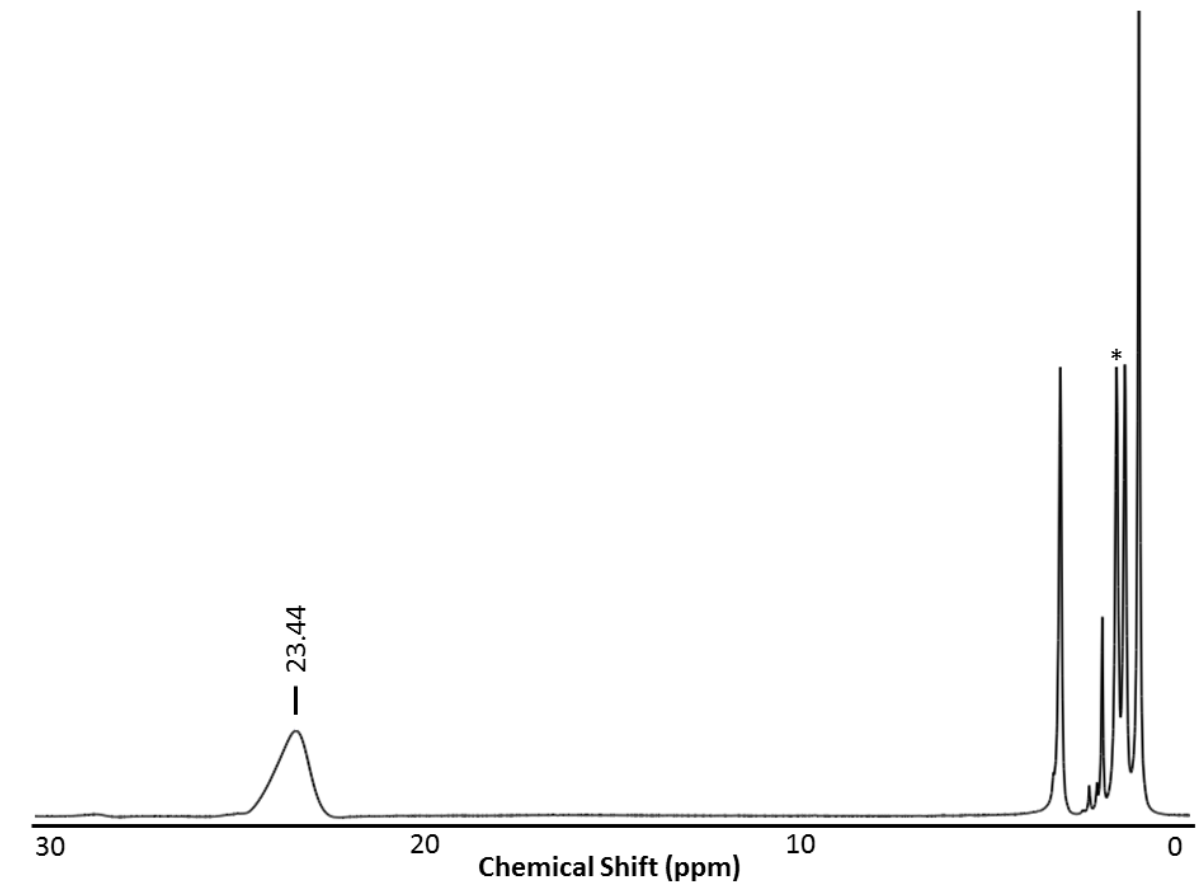

Figure S2a. ${ }^{1} \mathrm{H}$ NMR spectrum of complex $\left\{{ }^{\mathrm{n}} \mathrm{Bu}_{4} \mathbf{N}\right\}\left[\mathbf{V}_{6} \mathbf{O}_{7}\left(\mathbf{O C H}_{3}\right)_{12}\right]$ collected in $\mathrm{CD}_{3} \mathrm{CN}\left(21^{\circ} \mathrm{C}\right)$

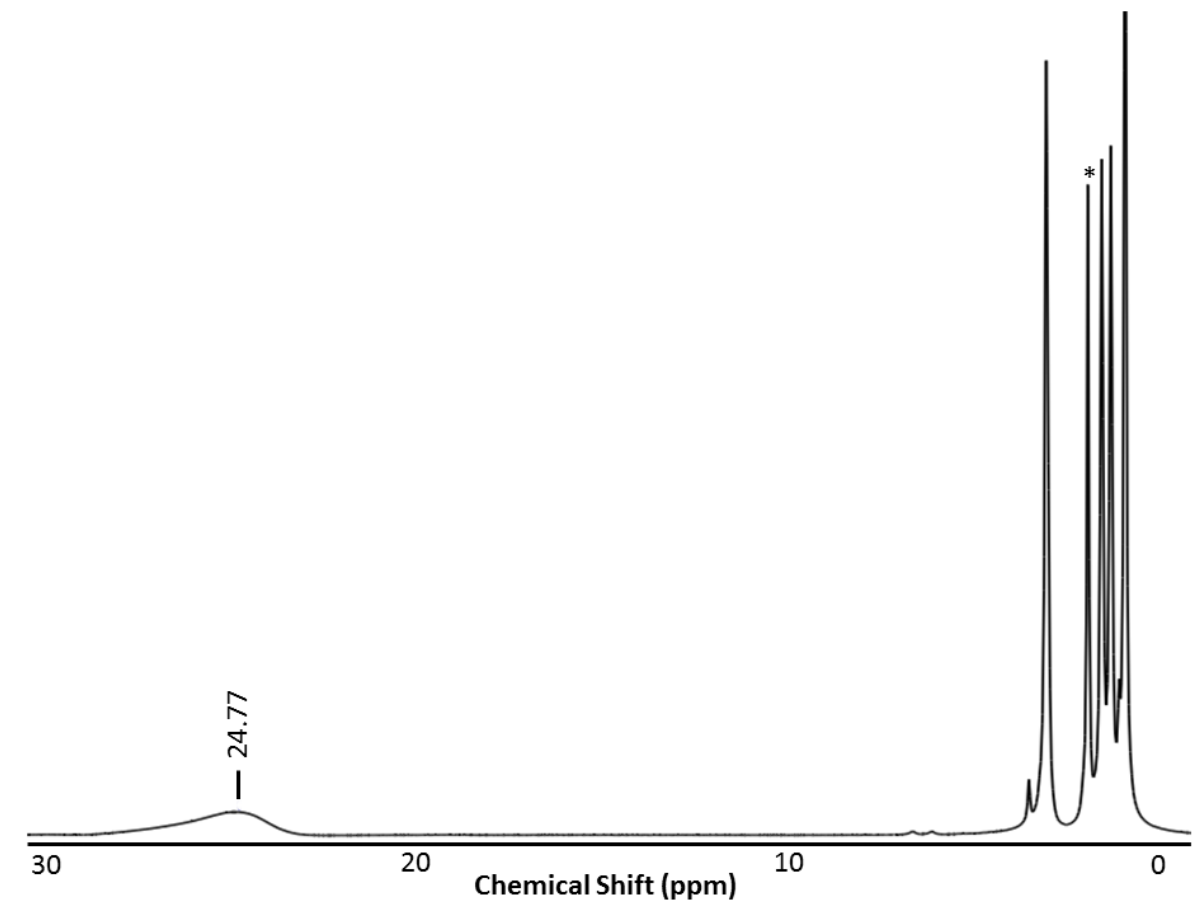

Figure S2b. ${ }^{1} \mathrm{H}$ NMR spectrum of cation exchange reaction of $\left\{{ }^{n} \mathbf{B u}_{4} \mathbf{N}\right\}\left[\mathbf{V}_{6} \mathbf{O}_{7}\left(\mathbf{O C H}_{3}\right)_{12}\right]$ with LiOTf collected in $\mathrm{CD}_{3} \mathrm{CN}\left(21^{\circ} \mathrm{C}\right)$ 


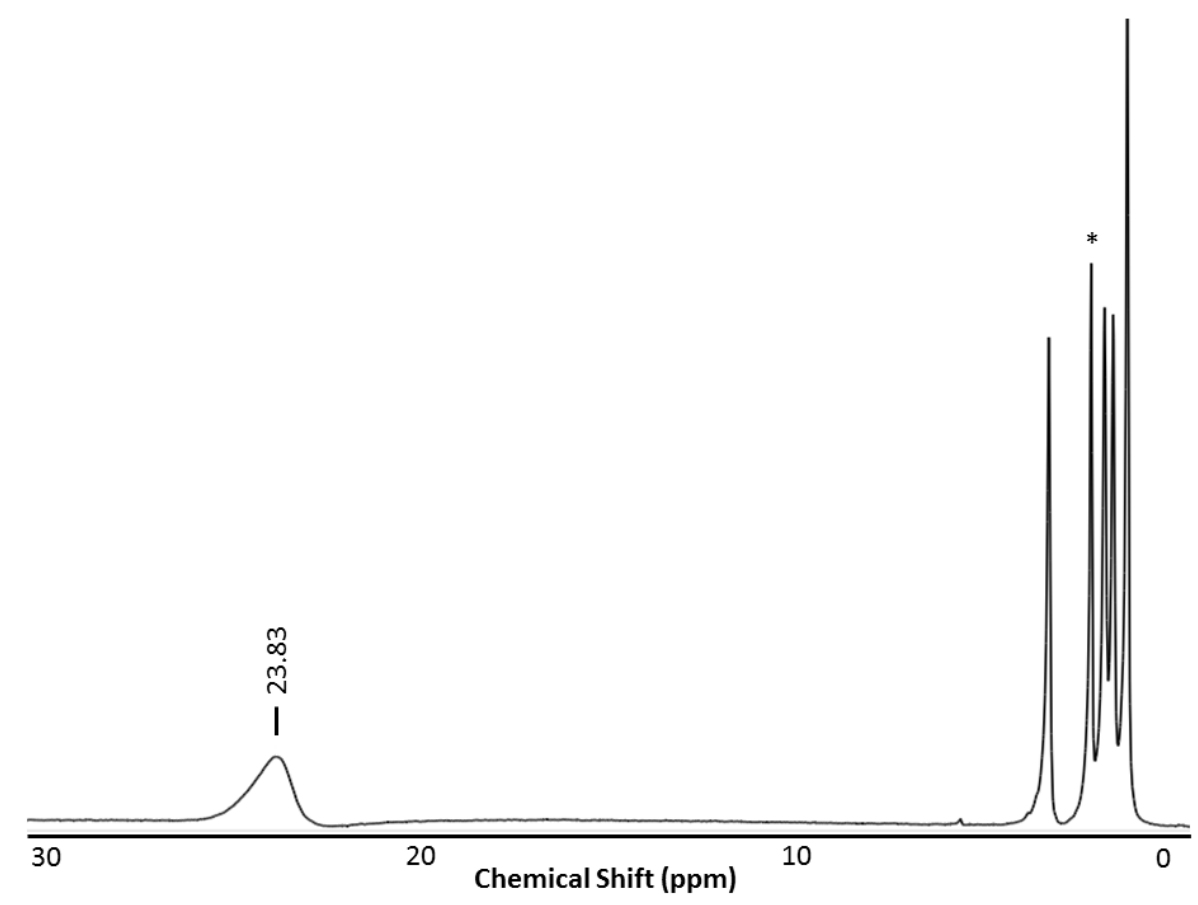

Figure S3a. ${ }^{1} \mathrm{H}$ NMR spectrum of complex $\left\{{ }^{n} \mathrm{Bu}_{4} \mathrm{~N}_{2}\left[\mathbf{V}_{6} \mathrm{O}_{7}\left(\mathrm{OCH}_{3}\right)_{12}\right]\right.$ collected in $\mathrm{CD}_{3} \mathrm{CN}\left(21^{\circ} \mathrm{C}\right)$

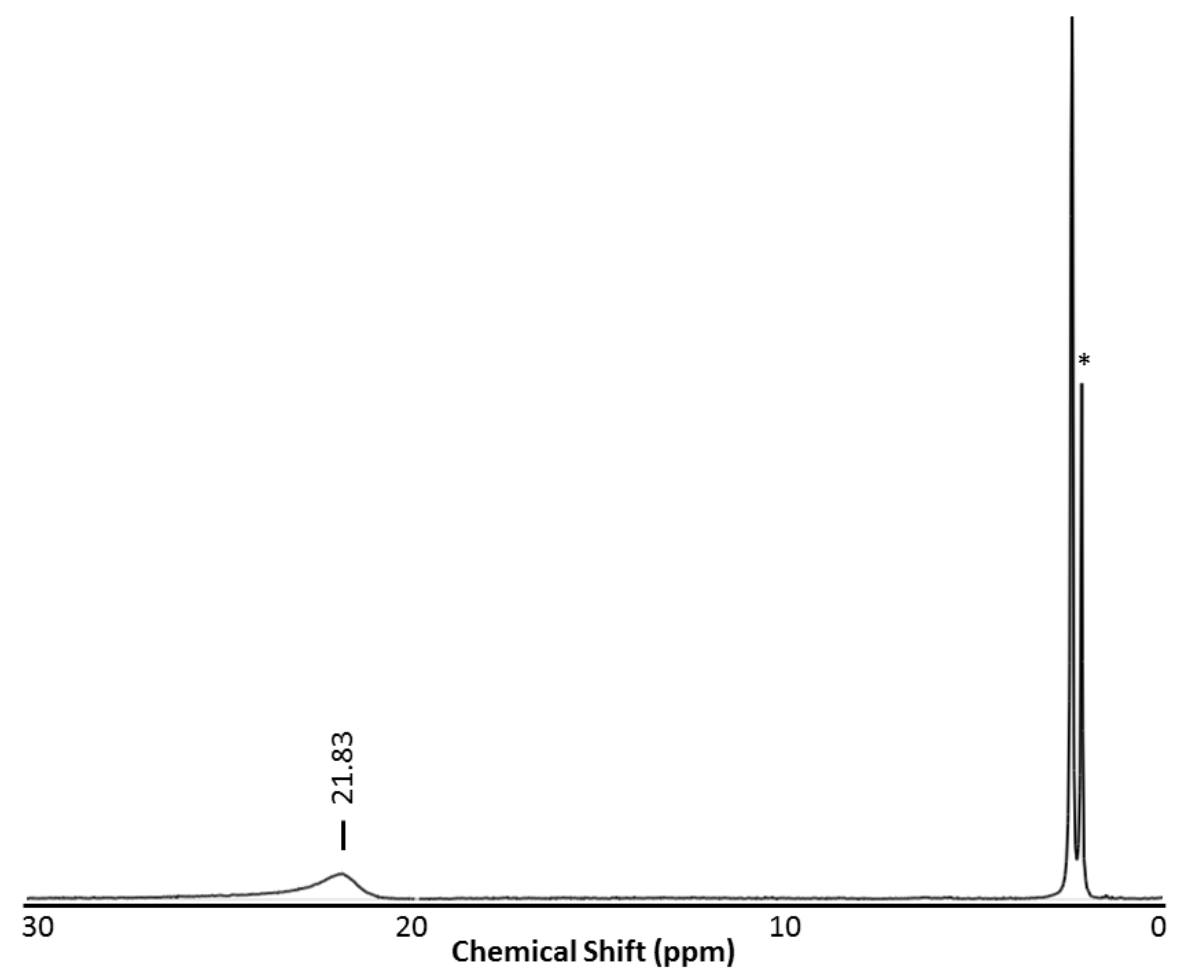

Figure S3b. ${ }^{1} \mathrm{H}$ NMR spectrum of complex $\{\mathrm{Li}\}_{2}\left[\mathbf{V}_{6} \mathbf{O}_{7}\left(\mathbf{O C H}_{3}\right)_{12}\right]$ collected in $\left(\mathrm{CD}_{3}\right)_{2} \mathrm{CO}\left(21^{\circ} \mathrm{C}\right)$ 
Table S3. Crystallographic parameters for complex $\{\mathrm{Li}\}_{2}\left[\mathrm{~V}_{6} \mathrm{O}_{7}\left(\mathrm{OCH}_{3}\right)_{12}\right]$.

\begin{tabular}{|c|c|c|}
\hline Empirical formula & \multicolumn{2}{|l|}{$\begin{array}{l}\mathrm{C}_{16} \mathrm{H}_{50} \mathrm{O}_{22} \mathrm{~V}_{6} \mathrm{Li}_{2} \\
(C C D C=1955968)\end{array}$} \\
\hline Formula weight & \multicolumn{2}{|l|}{914.08} \\
\hline Temperature & \multicolumn{2}{|l|}{$100.0(10) \mathrm{K}$} \\
\hline Wavelength & \multicolumn{2}{|l|}{$1.54184 \AA$} \\
\hline Crystal system & \multicolumn{2}{|l|}{ Monoclinic } \\
\hline Space group & \multicolumn{2}{|l|}{$P 2{ }_{1} / c$} \\
\hline Unit cell dimensions & $\begin{array}{l}a=17.4358(2) \AA \\
b=10.35010(10) \AA \\
c=20.6189(2) \AA\end{array}$ & $\begin{array}{l}\alpha=90^{\circ} \\
\beta=105.5010(10)^{\circ} \\
\gamma=90^{\circ}\end{array}$ \\
\hline Volume & \multicolumn{2}{|l|}{$3585.59(7) \AA^{3}$} \\
\hline Z & \multicolumn{2}{|l|}{4} \\
\hline Reflections collected & \multicolumn{2}{|l|}{36183} \\
\hline Independent reflections & \multicolumn{2}{|l|}{7544} \\
\hline Goodness-of-fit on F2 & \multicolumn{2}{|l|}{1.069} \\
\hline $\begin{array}{l}\text { Final } R \text { indices } \\
{[\mid>2 \text { sigma }(I)]}\end{array}$ & \multicolumn{2}{|c|}{$\mathrm{R} 1=0.0309, \mathrm{wR} 2=0.0757$} \\
\hline
\end{tabular}

\section{References.}

[1] Spandl, J.; Daniel, C.; Brüdgam, I.; Hartl, H. "Synthesis and Structural Characterization of RedoxActive Dodecamethoxoheptaoxohexavanadium Clusters" Angew. Chem., Int. Ed. 2003, 42, 1163-1166.

[2] Sheldrick, G. M. “SHELXT-2014/5” University of Göttingen, Göttingen, Germany, 2014.

[3] Sheldrick, G. M. "Crystal Structure Refinement with SHELXL" Acta Cryst. C. 2015, 71, 3-8. 